\title{
Long Term X-ray Variability of Galactic Nuclei
}

\author{
J. Cunniffe \& E.J.A. Meurs \\ Dunsink Observatory, Castleknock, Dublin 15, Republic of Ireland.
}

\begin{abstract}
We present some results on the long term variability of the $\mathrm{X}$-ray emission from optically catalogued galaxies, especially in view of their nuclear properties. For this investigation we use data from the ROSAT PSPC archive. Some future directions are also discussed.
\end{abstract}

\section{Introduction}

The ROSAT All Sky Survey (RASS) and subsequent pointed observations with the PSPC instrument have provided a database with a sufficient timebase to examine the long term variability of the X-ray emission from galactic nuclei. Many of the detected sources are recognised AGN but some will be galaxies which are not expected to have large X-ray brightness variations. NGC 5905, a proposed nuclear starburst galaxy (Bade, Komossa \& Dahlem 1996), was detected during the RASS in July 1990 but over subsequent observations its count rate dropped by a factor of approximately 100 taking it to the limit of detectability. This preliminary investigation aims to determine how many other galaxies which are not classified as AGN exhibit similar variations. Eventually, we hope to find candidates for variation in normal nuclei as has been suggested by the case of NGC 5905.

\section{Data}

An optical sample of galaxies was chosen from the CfA Redshift Catalogue (1995) (see Huchra et al. 1992) for those objects with type $T \leq 20$ and $M_{B}<13.21$ (the catalogue claims to be complete to this magnitude.)

These criteria produce 1774 objects which were compared with X-ray source detections from the Pointed Phase observations with the ROSAT PSPC instrument given in the WGA (Rev.1) Catalogue (White et al. 1994). Hardness ratios were defined as follows:

$$
H R 1=\frac{A-(B+C)}{A+B+C}, \quad H R 2=\frac{(A+B)-C}{A+B+C},
$$

where $A=P S P C$ channels 11 to $39, B=40$ to 84 , and $C=86$ to 200 . 


\section{Results}

Those optical positions matching to within 30" of the X-ray error circle (quoted by WGA as $13^{\prime \prime}$ within $20^{\prime}$ of PSPC axis, and 50" outside 20') are accepted as matches at this stage. This yields 237 objects with X-ray detections. Four of these objects are an alternative optical identification for the X-ray source and are thus rejected, leaving:

- $162 \mathrm{X}$-ray sources detected once (90 detected in a single pointing, 72 sources detected only once from multiple pointings.)

- $71 \mathrm{X}$-ray sources detected more than once.

At this point only the 71 sources with more than one X-ray detection will be discussed further as no variability information is directly available from the single detections. 3 sources are excluded as they contain X-ray detections which are too widely spread to be due to a single point source and a clear discrimination is not straightforward. The remaining objects are classified as active/non-active based on their inclusion/non-inclusion respectively as a Seyfert/QSO/BL Lac in the compilation of Véron-Cetty \& Véron (1998). Of these 68 objects, 30 are classified as active and 38 as non-active.

\section{Discussion}

The levels of variability of the PSPC count rate for all the sources here are relatively limited (certainly compared with the factor of 100 mentioned in the Introduction) with the highest factor being $\sim 7.5$ (active) and $\sim 3$ (non-active). $89 \%$ of non-active type galaxies have variabilities less than a factor of 2 , compared to $67 \%$ of active types. A greater level of variability may be seen when the 72 (out of 162) objects detected once from multiple pointings are analysed and upper limits calculated.

For non-active galaxies, the hardness ratios show little significant change with variation in count rate. Active galaxies, on the other hand, appear to show an overall trend towards greater softness at the lower count rate. However, this trend is absent in the few high variability cases in the sample of active types with little change in hardness ratio shown.

\section{References}

Bade,N., Komossa,S. \& Dahlem,M. 1996, A\&A, 309, L35

Huchra,J., Geller,M., Clemens,C., Tokarz,S. \& Michel,A. 1992, Bull. Inf. C.D.S. 41,31

Véron-Cetty \& Véron 1998, ESO Scientific Report 18

White,N.E., Giommi,P., Angelini,L. 1994, BAAS, 185 \#41.11 